\title{
Image Guided Transnasal Endoscople Fine Needle Aspiration or Blopsy of Parapharyngeal Space Tumours
}

\author{
Katharine Hamlett, Joshua Abbas, Jonathan Abbas, \\ John de Carpentier \\ Royal Preston Hospital, ENT Department
}

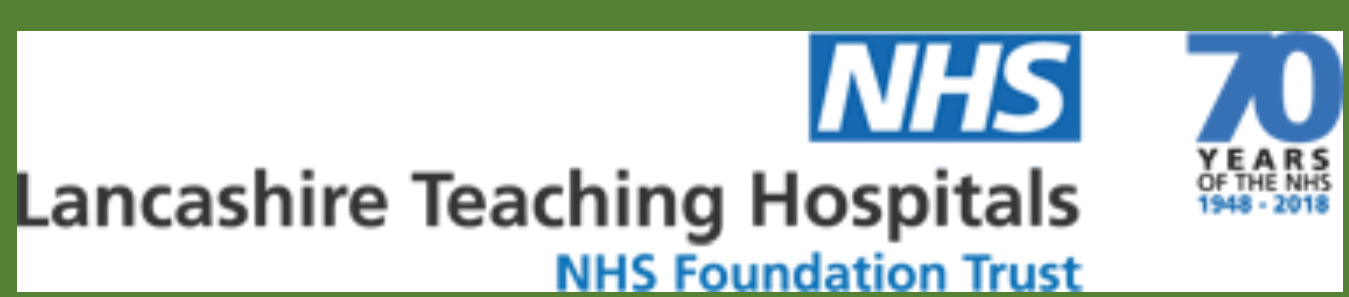

\section{Background}

The parapharyngeal space (PPS) is a potential space that lies lateral to the pharynx and deep to the palatine tonsils which is divided into the pre-styloid and post-styloid spaces by the fascia running from the styloid process to the tensor veli palatini muscle $^{1,2}$.

Lesions in the PPS have proven difficult to diagnose ${ }^{3-5}$, due to their deep location, inaccessibility and due to concerns about damaging important adjacent anatomical structures ${ }^{4,5}$.

The PPS biopsy is an important investigation in the management of PPS tumours. These tumours are relatively rare and the surgeon is often faced with a wide range of differential diagnoses.

We present a novel, less invasive, image guided transnasal endoscopic approach to PPS biopsy that has been successfully used by the senior author for years without any significant morbidity.

\section{Methods}

This technique is performed under general anaesthetic. We use Medtronic LandmarX Stealth ${ }^{\mathrm{TM}} \mathrm{CT}$ image guidance and preoperative scans need to be compatible with the LandmarX TM navigation system. The procedural steps are outlined below:

Step 1 - The patient is positioned supine with head up, similar to the positioning for functional endoscopic sinus surgery (FESS).

Step 2 - The LandmarX image guidance is set up as per protocol. The patient is registered and the equipment is calibrated.

Step 3 - The lesion is identified using the curved LandmarX probe. It is placed into the nose and angled laterally penetrating the mucosa and entering the PPS (figure 1). The image guidance assists the surgeon to decide trajectory and depth of penetration.

Step 4 - The sampling needle is usually a 22 gage $x 90 \mathrm{~mm}$ black spinal needle that is bent to exactly mirror the curve on the guidance probe. This is mounted onto a $10 \mathrm{ml}$ syringe.

Step 5 - The LandmarX probe is removed and the needle inserted into the pre-determined location. The syringe plunger is withdrawn to create negative pressure and the needle moved in and out, maintaining negative pressure to gain a satisfactory cytology sample.

Step 6 - The sample is injected into a pot of 10\% formalin and checked for adequacy. If more cells need to be obtained step 5 may be repeated. The sample is sent to the lab for cytology.

Step 7- The same procedure can be repeated using a curved "giraffe" forceps from the FESS set. The forceps are inserted in the closed position and opened within the tumour to obtain tissue samples that can be sent for histology.

\section{Discussion}

Masses in the PPS are relatively rare but do account for $0.5 \%$ of all masses arising in the head and neck ${ }^{1}$. Histological identification of the PPS tumour allows the surgeon to make informed decisions on management and can also dictate prognosis. The 5 and 10-year survival for a benign lesion is $100 \%$. Conversely, in malignancy the 5 -year survival drops to $82-100 \%$ and the 10 -year survival drops to 40-74\%. This dramatic fall in survival demonstrates a clear role for establishing the histology in the management of the PPS tumour.

There are a number of ways to access the PPS with some degree of morbidity, including needle biopsy through the mandibular or maxillofacial areas ${ }^{4}$, biopsy via the buccal space ${ }^{6}$, and directly through the skin or oral mucosa if the lesion is palpable ${ }^{5}$.

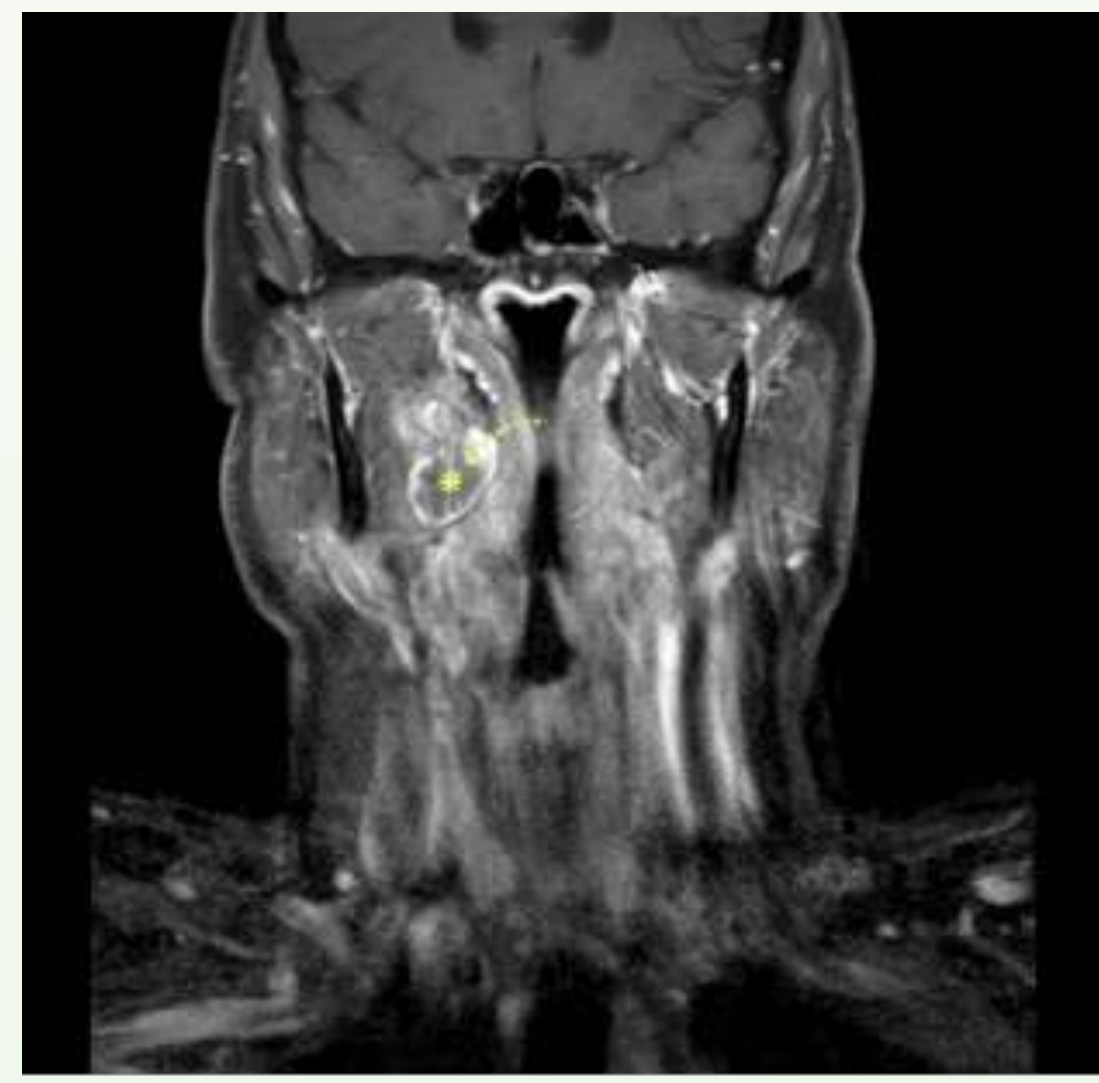

Figure 1: Coronal MRI neck, T1-weighted postgadolinium contrast. Star highlights lesion.

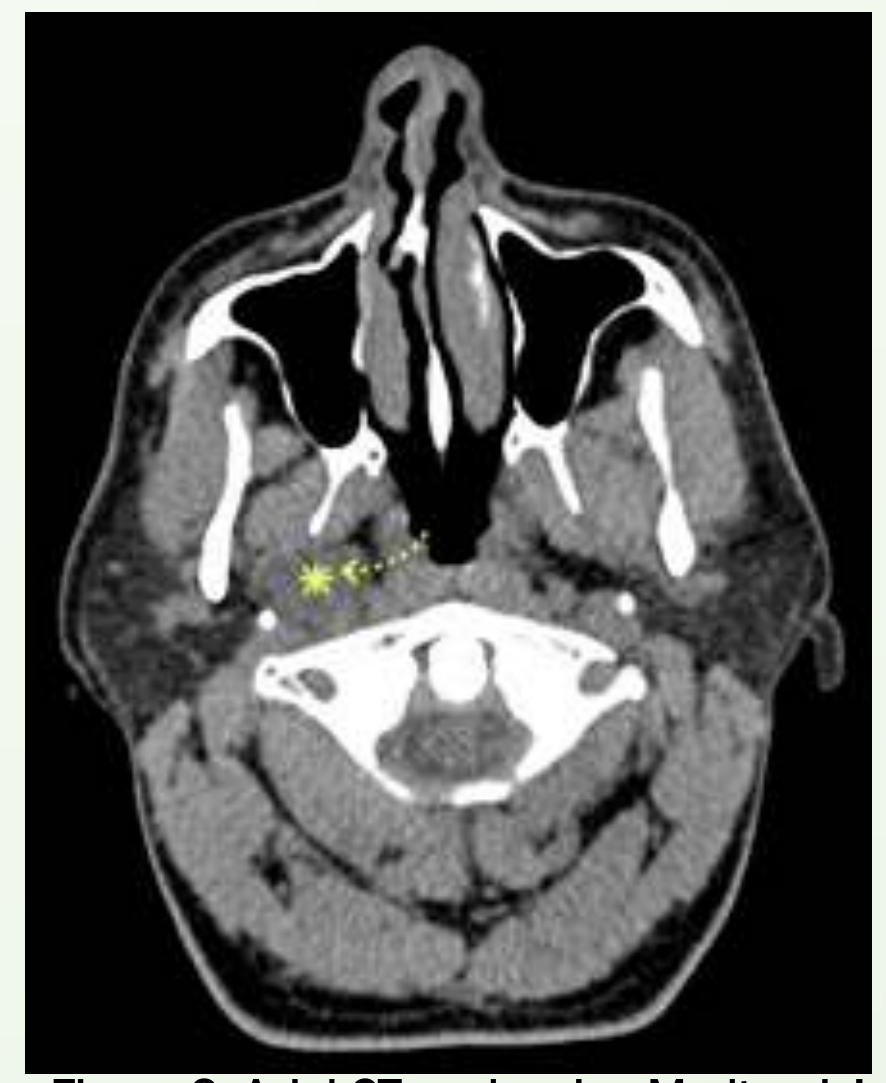

Figure 2: Axial CT neck using Medtronic's LandmarX $X^{\mathrm{TM}}$ navigation system.

\section{Key Points}

- PPS tumours are relatively rare

- The PPS is difficult to access given its proximity to important neck structures

- Current methods for PPS mass sampling are challenging \& potentially risky

- Transnasal access to the PPS is relatively simple in trained hands

- Peri-operative image guidance systems allow for accurate lesion targeting

\section{Conclusion}

Transnasal image guided endoscopic aspiration or biopsy of the PPS is a novel technique which has not been described previously. In trained hands, it has been shown to gain accurate results without complication.

We believe this procedure is relatively simple, safe and reproducible. The main disadvantage is the requirement of the LandmarX ${ }^{\mathrm{TM}}$ navigation system and the expertise required to use this equipment before attempting the procedure.

All the FNA or biopsy samples that have been performed via this technique thus far have been adequate for cytological or histological analysis. There have been no complications experienced in any of the patients undergoing this technique. 